\title{
Malaria Elimination in India: Bridging the Gap Between Control and Elimination
}

\author{
Shrikant Nema ${ }^{1}$, Pawan Ghanghoria ${ }^{2}$ and Praveen Kumar Bharti ${ }^{1}$ \\ From ${ }^{1}$ Division of Vector Borne Diseases, ICMR-National Institute of Research in Tribal Health; and ${ }^{2}$ Department of Pediatrics, \\ Netaji Subhash Chandra Bose Medical College; Jabalpur, Madhya Pradesh, India. \\ Correspondence to: Dr. Praveen Kumar Bharti, Scientist 'E', Division of Vector Borne Diseases, ICMR-National Institute of \\ Research in Tribal Health (NIRTH), DHR, Ministry of Health and Family Welfare, Government of India, Nagpur Road, PO - Garha, \\ Jabalpur 482 003, Madhya Pradesh, India.saprapbs@yahoo.co.in.
}

\begin{abstract}
India observed a significant reduction in malaria cases in the previous year, reaffirming our trust and efficiency of the existing tools to achieve malaria elimination. On 25 April, 2019, countries around the world marked World Malaria Day under the theme "Zero malaria starts with me". This provides an opportunity to rejoice the success and re-evaluate ongoing challenges in the fight against this preventable and treatable parasitic disease. We highlight the potential gaps in the malaria elimination program, and underscore potential solutions and strategies to implement, improve and intensify the success of the national goal of malaria elimination by 2030.
\end{abstract}

Keyword: Diagnosis, Epidemiology, Vector-borne disease.

I ndia has a long history of success and struggles with malaria control. The unsuccessful endeavor to eliminate malaria, and increasing morbidity and mortality bring back the elimination agenda in the health care priorities [1]. In 1976, there was a massive resurgence of malaria cases and Plasmodium falciparum resistance to chloroquine and vector resistance to insecticides were reported [1]. As a consequence, the modified plan of operations was launched in 1977 with a three-pronged strategy: early diagnosis with prompt treatment, vector control, and Information Education Communication (IEC)/Behavior Change Communication (BCC), resulting in the decline of malaria incidence again in 1984. Subsequently, Enhanced Malaria Control Project in 1997 and Intensified Malaria Control Project in 2005 were launched to combat malaria in high transmission areas of the country. New tools for malaria prevention and control were introduced by National Vector Borne Disease Control Program (NVBDCP) i.e., monovalent rapid diagnostic tests (RDT) for $P$. falciparum detection in 2005; Artemisinin-based combination therapy (ACT) in 2006; Long-lasting insecticide-treated nets (LLINs) in 2009; antigen detecting bivalent RDTs for detection of both $P$. falciparum and P. vivax in 2013; and newer insecticides and larvicides in 2014-15. However, these strategies failed to build on its expected level of achievements. India moved towards global commitment for malaria elimination and endorsed a plan to eliminate malaria throughout the region by 2030 [2]. World health organization (WHO) has developed the Global technical strategy for malaria under the National framework for malaria elimination in India 2016-2030 to eliminate malaria (zero indigenous cases) throughout the entire country by 2030 , and maintain malaria-free status and prevent its re-introduction. Therefore, we need to put all our efforts to achieve the desired success this time.

\section{DISEASE BURDEN AND SURVEILLANCE}

In 2018, an estimated 228 million cases of malaria occurred worldwide, compared to 251 million cases in 2010 [3]. In India, a population of 126 million was at risk of malaria with an estimate of 6 million cases in 2018 [3], while 0.43 million confirmed cases of malaria were reported by NVBDCP in 2018 [4]; although, discrepancies between various sources have been noted [5]. In India, malaria is highly endemic in rural and tribal areas of Madhya Pradesh, Maharashtra, Odisha, Rajasthan, Gujarat, Jharkhand, Chhattisgarh, Andhra Pradesh, West Bengal, and Karnataka. Further, districts with $30 \%$ or more tribal population comprising about $8 \%$ of the country's population contributed to $46 \%$ of total malaria cases, $70 \%$ P. falciparum cases and $47 \%$ malarial deaths in the country [6]. However, India has shown a $71 \%$ reduction in 2019 as compared to 2015 and this reduction was achieved by strengthening the surveillance measures, improving diagnosis and treatment, and intensive vector control measures using existing tools. For example, Odisha contributed $37.4 \%$ of total malaria cases in 2015 which reduced to $12 \%$ in 2019 using the Durgama 
Anchalare Malaria Nirakaran (DAMaN) initia-tive and compre-hensive case management of malaria. To sustain the achieved reduction and moving forward to the elimination, we have to strengthen all the strategies using existing tools and by developing new tools.

\section{CHALLENGES AND SOLUTIONS}

\section{Strengthening Malaria Diagnosis}

Accurate diagnosis is the key to success in the elimination goal. Among the five Plasmodium species, $P$. falciparum and $P$. vivax cause the majority of cases and other species are rare, but the diagnosis is complicated by the varied distribution of both mono-infection and mixed infections [7]. Microscopy has always been the gold standard method but it requires highly skilled microscopist with genuine knowledge of different stages of Plasmodium species with capability to read low-density parasitemia - fulfilling such a requirement in rural India is a daunting task, as a consequence, more than a quarter of malaria cases are missed by microscopy [8]. RDTs are used where microscopy is not feasible. $P$. falciparum histidine-rich protein 2 (PfHRP2) antigen targeting $P$. falciparum is used in more than $90 \%$ of the malaria RDTs [9]. However, deletions of the Pfhrp 2 gene in the parasite, fluctuation in the expression level of Plasmodium Lactic Dehydrogenase $(\mathrm{pLDH})$, and prozone phenomena are the major problems leading to inaccurate diagnosis of plasmodium species. Therefore, other potential biomarkers such as hemedetoxification protein, apical merozoites surface protein Pf34, Glutamate dehydrogenase, and hypnozoites-based serological marker should be validated to strengthen the RDT tool. Molecular methods such as Polymerase chain reaction (PCR) are feasible for the diagnosis of malaria (particularly low-density infection). However, these methods like conventional PCR, nested PCR, qPCR, multiplex PCR, and Loop-mediated isothermal amplification (LAMP) are less frequently used techniques due to longer time required, need for advanced equipment, expensive reagents and experienced personnel, and difficultly in organizing in most field conditions. A hemozoin-based magneto-optical detection device (Gazelle) may prove an alternative to RDT for accurate diagnosis in the field. These new markers/tools can make an impact on elimination efforts by addressing the problem of missed diagnosis.

\section{The Frontline Staff}

Accredited Social Health Activists (ASHA) and community health workers are the key players and leading contributors to the malaria elimination program as they are primary healthcare providers in the malaria endemics rural and tribal areas where government hospital and healthcare facilities are inaccessible. They provide diagnosis using RDT, and treatment, as well as advise them about the importance of preventive measures. Strengthening the qualitative and quantitative capacity of the ASHA may prove an asset in malaria elimination as children under the age of 5 are more vulnerable in the community for developing severe malaria. Tribal people are mostly dependent on traditional healers and unlicensed medical practitioners (UMP), which delays the correct diagnosis, and improper treatment may lead to severe malaria, as well as further transmission in the community [10]. Therefore, the stakeholders may think about providing training to unlicensed medical practitioners on national guidelines for malaria diagnosis and treatment to overcome this issue. An integrated community case management strategy along with ASHA/UMP may be needed to fight against malaria in the community.

\section{Malaria in Children}

Children aged below 5 years are the most vulnerable group and accounted for $67 \%$ of global malaria deaths in 2018 [3], and complicated malaria is more common in children than adults. The clinical symptoms (fever, vomiting, cough, difficulty in breathing and inability to eat and drink) of malaria in children may be mistaken for a viral syndrome or acute gastroenteritis. P. falciparum seems to be notorious for severe malaria but vivax is also presenting as severe malaria in children [11]. In high transmission areas, young children are at high risk of severe vivax-associated anemia, where the relapses phenomenon is frequent [12]. Children need portable, easy to take medicine adapted to their weight and age. Therefore, careful consideration should be given to the formulations of child-friendly antimalarials because children absorb and metabolize medicines differently [13]. Although the medicines for malaria ventures (MMV) has taken the initiative for discovering and developing new medicines [13]. The improper and inadequate drug and doses in the long term may create problems of drug resistance resulting in high morbidity and mortality in children as deaths in infants and children $<14$ years of age accounted for $20.6 \%$ in India [14].

\section{Pregnant Women: A Vulnerable Group}

Pregnant women are more susceptible to malaria, although the prevalence during pregnancy was substantially lower in areas of high transmission [15]. During placental malaria, $P$. falciparum-infected erythrocytes sequester in the placenta, causing health problems for both the mother and fetus, increasing risk for congenital malaria [16]. Therefore, in the malariaendemic areas, pregnant women should be screened for 
malaria if they have malaria-like symptoms or even in the cases of anemia, which not only helps malaria elimination but also delivering a healthy baby.

\section{Migration Malaria and Surveillance Strategy}

Migration malaria is also an important affair as it serves as a reservoir and seeds local outbreaks. Moreover, migrant workers who either take temporary shelter or coming from malaria-endemic areas could impede surveillance. Therefore, imported/migratory cases should be tracked by using surveillance networks, similar to GeoSentinel, EuroTravNet and TropNetEurop. Malaria elimination requires a strong surveillance mechanism that can reliably and rapidly detect the disease using the '1-37 ' strategy [17] and the ' $1-2-5$ ' strategy [18] during the elimination phase to overcome the problem. Additionally, mobile surveillance tools may be efficient in real-time information sharing such as Solutions for Community Health-workers $(\mathrm{SOCH})$ and Integrated Health Information Platform (IHIP) to prevent them from spreading disease and outbreak situations [19]. Therefore, the utilization of such networks may have importance in the malaria elimination program in India.

\section{Asymptomatic/ Afebrile Malaria: A Reservoir}

Afebrile cases do not show presentable routine symptoms but may become a source of parasitic transmission under a favorable setting. Asymptomatic malaria (the presence of sexual or asexual parasites and/or absence of clinical symptoms) poses a serious challenge worldwide [20]. Naturally acquired immunity and partial immunity with past exposure and age are the probable factors to asymptomatic malaria in the malaria-endemic areas that plays a significant role in transmission and malaria severity in children 2 to 5 years of age [21]. Therefore, proper attention is warranted in children; else they may act as a key reservoir of malaria infection.

\section{Antimalarial Drug Treatment and Resistance}

Schizonticidal and gametocidal drugs have been used to treat and prevent malaria for centuries. Chloroquine was first developed in the 1930s; but in 1973, chloroquineresistance (CQR) was initially pointed out in Assam, India. The rise in $\mathrm{CQR}$ ( $P f_{c}$ rt gene, a molecular marker to track the CQR) contributed to a worldwide increase in malaria-related mortality. To combat resistant strains, several alternative synthetic antimalarial drugs (sulfadoxine-pyrimethamine and mefloquine) were deployed to treat and prevent malaria. Sulfadoxinepyrimethamine (SP) is utilized as the second line of therapy after chloroquine-resistant in India. However, the mutation at the dhps and $d h f r$ genes make it ineffective against the $P$. falciparum malaria. The introduction of
ACT has made a thrilling effect on malaria treatment in many countries. At present, these drugs are successful; however, there are already hints that resistance to artemisinin has emerged [22]. Other factors that may contribute to drug resistance are the mutation in resistance markers, counterfeit or substandard treatments, improper doses, and artemisinin monotherapy. To avoid artemisinin resistance, triple artemisinin-based combination therapies such as artemether-lumefantrine plus amodiaquine are already in pipeline for the treatment of uncomplicated $P$. falciparum malaria [23]. In the case of $P$. vivax, a 14-day course of primaquine (gametocidal drug) is recommended in all transmission settings to overcome the issue of relapse but poor drug compliance is a major challenge.

However, the single dose regimen of tafenoquine may be helpful to improve the adherence issues associated with primaquine regimens. Clinicians must document the G6PD status because primaquine and tafenoquine both may induce hemolytic anemia in patients with a glucose6-phosphate dehydrogenase deficiency (G6PD). Nevertheless, novel $P$. vivax anti-relapse medicines that targets hypnozoites are greatly needed. Implementation of Directly-observed therapy (DOT) reduces the antimalarial resistance development, reappearance rate of the parasite, and may subsequently decrease $P$. vivax transmission [24]. This ultimate goal of developing new antimalarial drugs and modifying existing ones will take us one step closer to the elimination goal.

\section{Plasmodium vivax: Roadblock in the Success}

In India, $P$. vivax contributed $53.4 \%$ of the infections in 2019. It is often termed benign malaria but substantial increases in morbidity and mortality, especially in infants due to weak immunity is considered alarming. Pathophysiology of $P$. vivax such as a low-density bloodstage infection, hypnozoites, transmission facilitated by the early production of infective stages, mature gametocytes and more genetically diverse $P$. vivax populations have limited understanding. Vivax Duffynegative phenotype and Fy glycoprotein (FYA) need proper understanding in the Indian context to fight against $P$. vivax malaria [25].

The risk of $P$. vivax parasitaemia is high in coendemic regions (where both $P$. falciparum and $P$. vivax are equally prevalent) after treatment for $P$ falciparum infection. This is probably due to fast acting and rapid parasite clearance property of artemisinin-based therapy against the treatment of falciparum malaria, in the area where short periodicity of $P$. vivax relapse cases occurred. Therefore, complete radical cure may be assured to prevent recurrent parasitaemia, reduce ongoing 
transmission to ensure malaria elimination success [26].

\section{Vector Control and Insecticide Resistance}

National Malaria Program has distributed about 50 million Long Lasting Insecticidal nets (LLINs) to communities during 2016-2018 in India [4] as an intervention tool for malaria control and prevention to cover the 126 million populations that were under risk [3]. Among children under 5 years of age, LLINs provide up to $55 \%$ protective efficacy in preventing malaria attributed to mortality [27]. Operational success can only be achieved when universal coverage is attained and is at least $80 \%$ [28]. The major drawbacks of LLINs include personal discomfort and feelings of suffocation when humidity and indoor temperature are high. Therefore, child-friendly nets using color combinations and cartoon-based print may increase the use of LLIN. Thus, Zero vector durable lining (ZVDL) is designed to cover interior wall surfaces, utilizing slow-release technology that has the advantages of both LLINs and IRS, i.e. long-lasting residual use and no insecticide dusting [29].

\section{Vaccines: The Last Piece of the Puzzle}

There is no commercially available malaria vaccine currently. However, efforts to make an effective malaria vaccine are underway for the last three decades. Phase 3 trial of RTS, S/AS01 (Mosquirix) (at month 0, 1, and 2) in children aged 5-17 months showed vaccine efficacy of 28.3\% against severe malaria in children [30]. PfSPZ based genetically attenuated vaccines which halt the development in the early liver stages were found to offer protection to 55\% recepients [31]. Limited understanding of how immunity develops against malaria poses a great challenge to researchers in designing effective vaccines. Recent advances in the generation of recombinant proteins, DNA and RNA based approaches may be useful in vaccine development [32].

\section{Inter-sectoral Coordination}

Several government organizations, such as the ICMR through Malaria Elimination Research Alliance-India and NVBDCP are moving forward to fill the gaps with research and innovative strategies. India Health Fund and several non-governmental organi-zations such as TATA Trust and Godrej have also taken an initiative to work on parasite control, vector control, technology-integration, and awareness and behavioral change. Moreover, the success story of neighboring countries like Sri Lanka and China echoes the importance of public-private partnerships to accelerate malaria elimination efforts. ICMR and Sun Pharma Ltd have partnered for malaria elimination activities in Mandla district of Madhya Pradesh [19].

INDIAN PEDIATRICS

\section{CONCLUSION}

Healthcare communities have undertook serious efforts to reduce malaria cases in India, but it is still threatening millions in India. This time the elimination efforts would require targeted approaches and strategies starting from the village level to the national level. At the same time, we need to take care of all the possible gaps such as human resources, robust surveillance, and hotspot targeted interventions by proper utilization of existing as well as new tools. All the laboratory-confirmed positive cases should be advised to stay under mosquito net until parasite clearance to avoid community transmission. Universal and continuous availability of drugs, diagnostic and essential malaria commodities are to be ensured for effective management of community malaria. If the lessons learned from elimination efforts are properly utilized, the malaria elimination goal mayvery well be achieved on time.

Contributors: All authors have contributed, drafted and approved the manuscript.

Funding: None; Competing interests: None stated.

\section{REFERENCES}

1. Sharma VP. Re-emergence of malaria in India. Ind J Med Res. 1996;103:26-45.

2. Guerin PJ, Dhorda M, Ganguly NK, Sibley CH. Malaria control in India: A national perspective in a regional and global fight to eliminate malaria. J Vector Borne Dis. 2019; 56:41-5.

3. World Health Organization. World Malaria Report 2019. Available from: https://www.who.int/publications-detail/ world-malaria-report--2019. Accessed December 7, 2019.

4. National Vector Borne Disease Control Programme (NVBDCP). Malaria. Available from: https://nvbdcp.gov. in/index1.php? lang $=1$ \&level $=1 \&$ sublinkid $=5784 \&$ lid $=$ 3689. Accessed October 8, 2019.

5. Dhingra N, Jha P, Sharma VP, Cohen AA, Jotkar RM, Rodriguez PS, et al., Million Death Study Collaborators. Adult and child malaria mortality in India: A nationally representative mortality survey. Lancet. 2010;376:1768-74.

6. Sharma RK, Thakor HG, Saha KB, Sonal GS, Dhariwal AC, Singh N. Malaria situation in India with special reference to tribal areas. Indian J Med Res. 2015;141:537-41.

7. Nema S, Verma AK, Bharti PK. Strengthening diagnosis is key to eliminating malaria in India. Lancet Infect Dis. 2019;19:1277-8.

8. Okell LC, Ghani AC, Lyons E, Drakeley CJ. Submicroscopic infection in Plasmodium falciparumendemic populations: a systematic review and metaanalysis. J Infect Dis. 2009;200:1509-17.

9. Ahmad A, Verma AK, Krishna S, Sharma A, Singh N, Bharti PK. Plasmodium falciparum glutamate dehydrogenase is genetically conserved across eight malaria endemic states of India: Exploring new avenues of malaria elimination. PloS One. 2019;14:e0218210. 
10. Singh MP, Chand SK, Saha KB, Singh N, Dhiman RC, Sabin LL. Unlicensed medical practitioners in tribal dominated rural areas of central India: Bottleneck in malaria elimination. Malaria J. 2020;19:18.

11. Singh J, Purohit B, Desai A, Savardekar L, Shanbag P, Kshirsagar N. Clinical manifestations, treatment, and outcome of hospitalized patients with Plasmodium vivax malaria in two Indian states: a retrospective study. Malar Res Treat. 2013;2013:341862.

12 Douglas NM, Anstey NM, Buffet PA, Poespoprodjo JR, Yeo TW, White NJ, Price RN. The anaemia of Plasmodium vivax malaria. Malaria J. 2012;11:135.

13. Medicines for Malaria Venture. Children and malaria: treating and protecting the most vulnerable. Available from:https://www.mmv.org/newsroom/publications/ children-and-malaria-treating-and-protecting-mostvulnerable. Accessed December 8, 2019.

14. Kumar A, Valecha N, Jain T, Dash AP. Burden of malaria in India: Retrospective and prospective view. Am J Trop Med Hyg. 2007;77(6_Suppl): 69-78.

15. Kitojo C, Gutman JR, Chacky F, Kigadye E, Mkude S, Mandike R, et al. Estimating malaria burden among pregnant women using data from antenatal care centres in Tanzania: a population-based study. Lancet Glob Hlth. 2019;7:e1695-705.

16. Pereira MA, Clausen TM, Pehrson C, Mao Y, Resende M, Daugaard M, et al. Placental sequestration of Plasmodium falciparum malaria parasites is mediated by the interaction between VAR2CSA and chondroitin sulfate A on syndecan-1. PLoS Pathog. 2016;12:e1005831.

17. Zhou SS, Zhang SS, Zhang L, Rietveld AE, Ramsay AR, Zachariah R, et al. China's 1-3-7 surveillance and response strategy for malaria elimination: Is case reporting, investigation and foci response happening according to plan? Infect Dis Poverty. 2015;4:55.

18. Sitohang V, Sariwati E, Fajariyani SB, Hwang D, Kurnia B, Hapsari RK, et al. Malaria elimination in Indonesia: Halfway there. Lancet Global Hlth. 2018;6: e604-6.

19. Lal AA, Rajvanshi H, Jayswar H, Das A, Bharti PK. Malaria elimination: Using past and present experience to make malaria-free India by 2030 . J Vector Borne Dis. 2019; 56:60-5.

20. Hassanpour G, Mohebali M, Zeraati H, Raeisi A, Keshavarz H. Asymptomatic malaria and its challenges in the malaria elimination program in Iran: A systematic review. J Arthropod Borne Dis. 2017;11:172-4.

21. Cheaveau J, Mogollon DC, Mohon MA, Golassa L,
Yewhalaw D, Pillai DR. Asymptomatic malaria in the clinical and public health context. Expert Rev Anti-infect Ther. 2019;17:997-1010.

22. Das S, Saha B, Hati AK, Roy S. Evidence of artemisininresistant Plasmodium falciparum malaria in eastern India. New Engl J Med. 2018;379:1962-4.

23. van der Pluijm RW, Tripura R, Hoglund RM, Phyo AP, Lek D, Ul Islam A, et al. Triple artemisinin-based combination therapies versus artemisinin-based combination therapies for uncomplicated Plasmodium falciparum malaria: a multicentre, open-label, randomised clinical trial. Lancet. 2020 395:1345-60.

24. Takeuchi R, Lawpoolsri S, Imwong M, Kobayashi J, Kaewkungwal J, Pukrittayakamee S, et al. Directlyobserved therapy (DOT) for the radical 14-day primaquine treatment of Plasmodium vivax malaria on the ThaiMyanmar border. Malaria J. 2010;9:308.

25. Gai PP, Van Loon W, Siegert K, Wedam J, Kulkarni SS, Rasalkar R, et al. Duffy antigen receptor for chemokines gene polymorphisms and malaria in Mangaluru, India. Malaria J. 2019;18:328.

26. Commons RJ, Simpson JA, Thriemer K, Hossain MS, Douglas NM, Humphreys GS, et al. Risk of Plasmodium vivax parasitaemia after Plasmodium falciparum infection: a systematic review and meta-analysis. Lancet Infect Dis. 2019;19:91-101.

27. Eisele TP, Larsen D, Steketee RW. Protective efficacy of interventions for preventing malaria mortality in children in Plasmodium falciparum endemic areas. Int J Epidemiol. 2010;39(suppl_1):i88-101.

28. Walldorf JA, Cohee LM, Coalson JE, Bauleni A, Nkanaunena K, Kapito-Tembo A, et al. School-age children are a reservoir of malaria infection in Malawi. PloS One. 2015;10: e0134061.

29. Mishra AK, Bharti PK, Kareemi TI, Chand SK, Tidgam AS, Sharma RK, et al. Field evaluation of zero vector durable lining to assess its efficacy against malaria vectors and malaria transmission in tribal areas of the Balaghat district of central India. Trans Royal Soc Trop Med Hyg. 2019;113:623-31.

30. Lancet T. Vaccines: A step change in malaria prevention? Lancet. 2015;385:1591-3.

31. Ghosh SK, Rahi M. Malaria elimination in India - the way forward. J Vector Borne Dis. 2019;56:32-40.

32. Doolan DL, Hoffman SL. DNA-based vaccines against malaria: status and promise of the multi-stage malaria DNA vaccine operation. Int J Parasitol. 2001;31:753-62. 